\title{
LOW BACK PAIN, THE STIFFNESS OF THE SACROILIAC JOINT: A NEW METHOD USING ULTRASOUND
}

\author{
E. Vlaanderen, ${ }^{*}$ N. E. Conza, ${ }^{\ddagger}$ C. J. Snijders, ${ }^{*}$ A. Bouakaz, ${ }^{\dagger}$ and N. De Jong ${ }^{\dagger}$ \\ *Biomedical Physics and Technology and ${ }^{\dagger}$ Experimental Echocardiography, Erasmus Medical Center, Rotterdam, \\ The Netherlands; and Faculty of Mechanical Engineering and Marine Technology, Engineering Mechanics- \\ Dynamics, University of Technology, Delft, The Netherlands
}

(Received 24 May 2004; revised 23 September 2004; accepted 30 September 2004)

\begin{abstract}
Abnormal biomechanical properties of the sacroiliac joints are believed to be related to low back and pelvic pain. Presently, physiotherapists judge the condition of the sacroiliac joints by function and provocation tests, and palpation. No objective measuring device is available. Research is ongoing to identify the biomechanical properties of the sacroiliac joints from the dynamic behaviour of the pelvic bones. A new concept based on ultrasound (US) for the measurement of bone vibration is under investigation. The objective of this study was to validate this concept on a physical model and to assess the applicability in vivo. A model consisting of a piezo shaker covered by a layer of US transmission gel (representing bone and soft tissue) has been used. A packet of US detection signals is directed onto the shaker and correlation-based processing is used to estimate the difference in time-of-flight of their echoes. These variations of time are used to compute the displacement of the shaker at each pulse reflection. To assess the validity of our US technique, we compared the obtained measurements with the readings of the built-in strain gauge sensor. The experimental procedure has been tested on a volunteer where low-frequency excitation was provided through the ilium and vibration detected on the sacrum and ilia. The results demonstrated that the correlation-based approach is capable of reproducing the piezo shaker displacements with high accuracy $( \pm 7 \%)$. Vibration amplitudes from $0.25 \mu \mathrm{m}$ to $3 \mu \mathrm{m}$ could be measured. The US technique was able to detect bone vibration in vivo. In conclusion, the principle based on US waves can be used to develop a new measurement tool, instrumental in studying the relation between the biomechanical properties of the sacroiliac joints and low back pain. (E-mail: e.vlaanderen@erasmusmc.nl) @ 2005 World Federation for Ultrasound in Medicine \& Biology.
\end{abstract}

Key Words: Ultrasound, Vibrations, Low back pain, Sacroiliac joint.

\section{INTRODUCTION}

Low back pain is one of the major causes of discomfort in the world. The origin of low back pain is the focal point of many investigations: in some cases, pathology can be identified during medical screening but, in most other cases, there is no medical evidence of the cause of the complaints (Kovacs et al. 2004; Mofidi et al. 2003).

Abnormal biomechanical properties of the sacroiliac joints are considered as a potential source of low back pain (Pool-Goudzwaard et al. 2003; Sims and Moorman 1996; Snijders et al. 1993). Function tests, provocation tests and palpation are among the techniques that are frequently used in physiotherapy and orthopaedic ther-

Address correspondence to: Dr. Ester Vlaanderen, Erasmus MC, Biomedical Physics and Technology, Ee1683, P. O. Box 1738, Rotterdam 3000 DR The Netherlands. E-mail: e.vlaanderen@erasmusmc.nl apy to obtain information on possible manifestation of these joint abnormalities (hypermobility or hypomobility) (Bickley and Szilagyi 2002; Greene and Heckman 1994; Hoppenfeld 1976). However, in specific joints, such as the sacroiliac, carpal and tarsal joints, these clinical examinations are impaired by limited excursion of the joints and by restricted accessibility to the regionof-interest (ROI). To describe these movements in the sacroiliac joints under different static loads, an x-ray technique as well as a conventional light photography technique have previously been explored (Jacob and Kissling 1995; Sturesson et al. 1989). In the x-ray technique, radiopaque markers can be inserted into the sacrum and ilium, and displacement of the markers after joint manipulation is measured on $\mathrm{x}$-ray photographs. In the conventional photography technique, external markers are introduced percutaneously. During different physiological exercises, rotations and translations of the ilium 


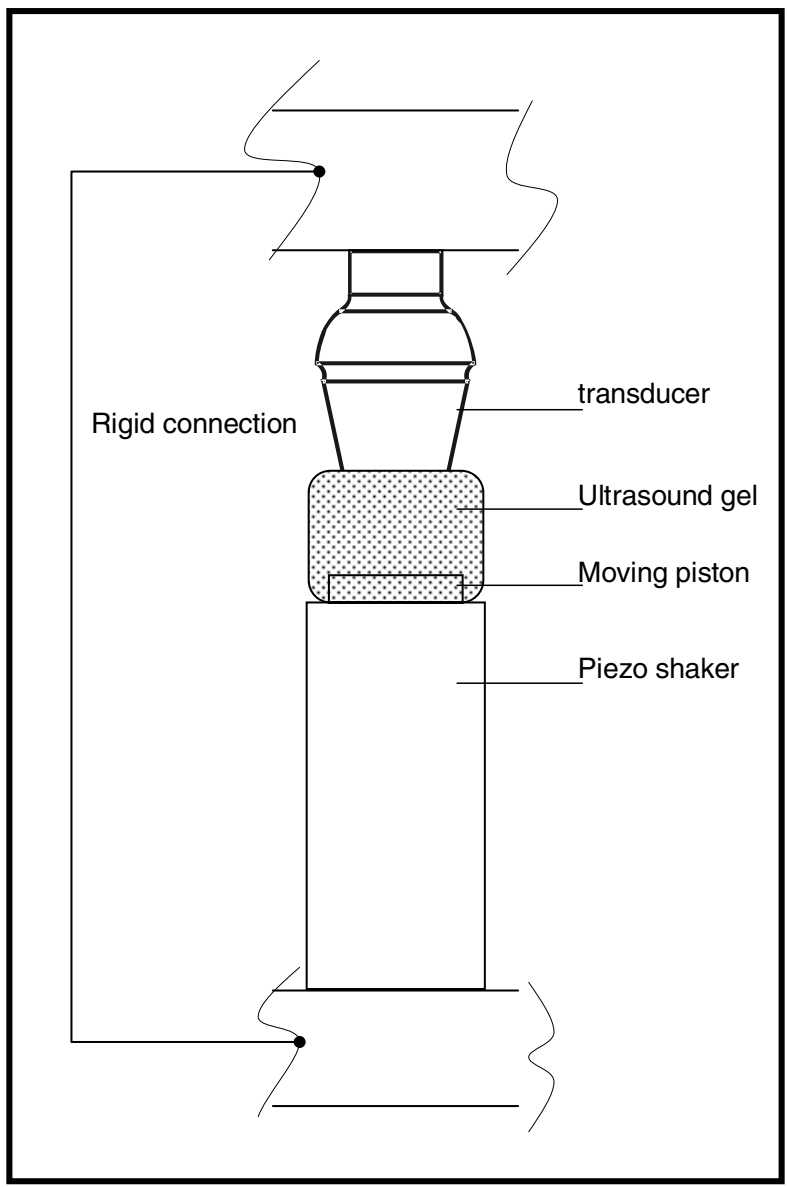

Fig. 1. A physical model consisting of a piezo shaker covered by a layer of US transmission gel represents a layered structure of bone and tissue.

in relation to the sacrum can be measured using either of these techniques.

However, there are several problems associated with the techniques described above for joint mobility assessment. In function and provocation tests, neither the applied stimulus nor the resulting response can be quantified. The diagnosis depends upon the perception and experience of the clinician. Function and provocation tests are, therefore, highly subjective and their diagnostic value is generally quite low, particularly in joints with limited excursions (Dreyfuss et al. 1996; van der Wurff et al. 2000a, 2000b). In addition, any diagnostic procedure using $\mathrm{X}$ rays is subject to the well-established problems, hazards and restrictions of such techniques, and can cause secondary effects to the subjects under examination. The positioning of markers for assessment of range of motion in joints with limited accessibility requires at least minimally invasive procedures, which increase the costs and risks of the procedures and discomfort to the patient.

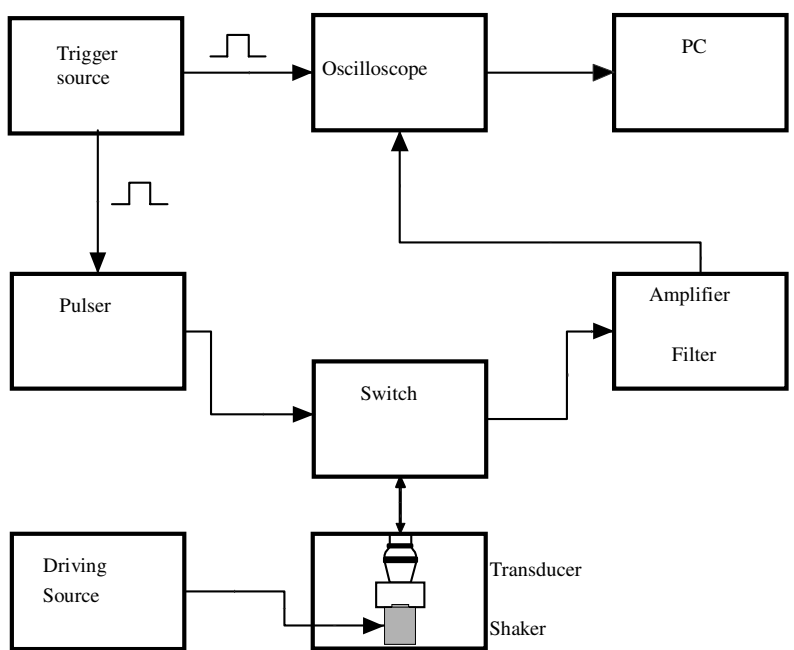

Fig. 2. Schematic representation of the experimental in vitro US setup.

Moreover, all the methods used to determine joint movements discussed above are only suitable for the determination of the static response of the joint. One important source of information for the determination of biomechanical properties is the dynamic response of the bones. For in vivo conditions, this dynamic response is expected to be of much lower amplitude (in the range of micrometres), than in a static test (typically in the order of a few millimetres) (Sturesson et al. 1989). The methods of measurement of static response are not sufficiently sensitive to measure displacements in the lower order of magnitude required for dynamic response.

Measurement of minimal movements or displacements of structures in the human body is a common issue in the medical field; estimation of blood flow velocity (Ferrara and DeAngelis 1997), tissue motion, and elasticity (Ophir et al. 1991) are based on the estimation of displacement through echo time-delay analysis. A recent technology that takes advantage of time-delay estimations is intravascular ultrasound (US) elastography (de Korte et al. 2003). In this technique, local strain in the artery wall and plaques is assessed by looking at the deformation of the tissue. Snijders et al. (1993) proposed mechanical excitation of the pelvic girdle to assess joint laxity, whereas Buyruk et al. (1995) introduced an US technique to measure the induced vibrations of the pelvic bones. These measurements were used to categorise laxity of the pelvic joints. The subject was examined while lying in a prone position on a physiotherapy table. The pelvis was unilaterally supported by a vibrating surface, located at the spina iliaca anterior superior (SIAS). This vibrating surface transmits vibrations to the ilium. To pick up the vibrations of the bones, a linear-array transducer was positioned over the dorsal side of the sacroil- 
iac joint. Detection of the vibrations was performed by means of an US scanner operating in colour Doppler imaging mode. The transmission of the vibrations from the ilium to the sacrum was believed to be deducible from the obtained colour flow map and was then used to rank the laxity of the intermediate sacroiliac joint. However, vibration parameters that characterise the dynamic response of the bones lie outside the range that can be quantified using this method. In addition, the correlation between the colour maps obtained and the actual vibration of the pelvis was not validated.

A further development in the investigation described above was to introduce a computer model of the human pelvis for a quantitative interpretation of the measured vibration. Unlike the work of Buyruk and colleagues, in this approach, the identification of the biomechanical properties of the sacroiliac joints (i.e., stiffness and damping) is based on a mathematical algorithm that uses the measured vibrations as an input (Conza and Rixen 2004). Relevant information in the dynamic response is expected within the frequency range of 10 up to $500 \mathrm{~Hz}$ and amplitudes in the interval of 0.1 up to $100 \mu \mathrm{m}$. The overall goal of the investigation is to develop an appropriate laboratory setup to be downscaled further to equipment that can be used in daily practice of physiotherapy, orthopaedics and rehabilitation medicine.

One of the challenges of the investigation described above is the noninvasive measurements of the vibration of the pelvic bones. Instead of exploiting the frequency shift of the returning US waves (Doppler effect), in our approach we base the measurement of the bone vibration on the time-delay estimation of the US waves. Although theoretically solid, the principle has to be verified and validated in practice by a series of experiments where the measurements obtained are compared to calibrated reference values.

The aim of this study is to validate the proposed US technique within the relevant working range and to demonstrate the feasibility of the experimental approach in vivo.

\section{MATERIALS AND METHODS}

A physical model (Fig. 1) consisting of a piezo shaker (P-841, Physik Instruments (PI) GmbH \& Co., KG, Karlsruhe/Palmbach, Germany) surmounted by a layer of US transmission gel represents a layered structure of bone and tissue. The piezo shaker, equipped with a strain gauge position sensor was operated in a closed loop. The control input was sinusoidal with preset frequencies of 80, 180 and $280 \mathrm{~Hz}$ and amplitudes from 250 $\mathrm{nm}$ up to $3 \mu \mathrm{m}$. An US transducer (Aerotech, Agfa, Rijswijk, the Netherlands) was positioned over the layer

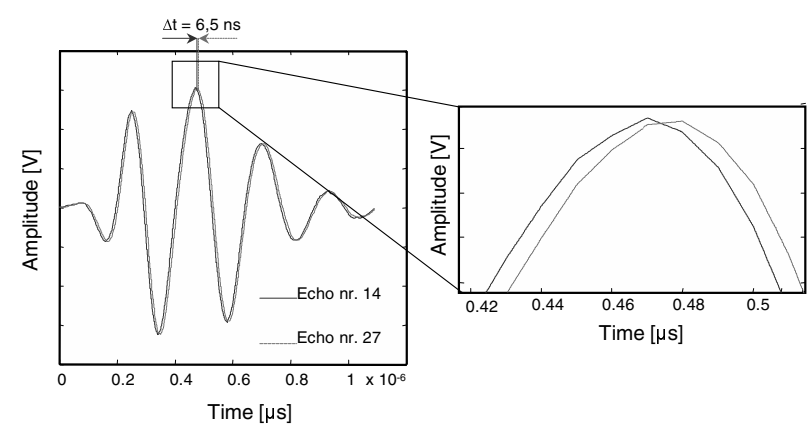

Fig. 3. The time delay between two recorded echoes resulting from a shaker vibrating at $180 \mathrm{~Hz}$ and $2.5 \mu \mathrm{m}$ amplitude.

of US gel, above the moving piston, and rigidly connected to the housing of the shaker. The rigid connection prevents movement of the transducer relative to the shaker.

\section{In vitro setup}

Figure 2 shows a schematic representation of the US setup. A single-element US transducer operating at $5 \mathrm{MHz}$ was used to measure the amplitude and frequency of the target (piezo shaker) vibration by emitting 62 pulses at a pulse repetition rate (PRR) of $5 \mathrm{kHz}$. The pulser and sampling oscilloscope were phase-synchronised using the external clock output of the digital oscilloscope and externally triggered by means of a pulse generator (PM 5712, Philips, The Netherlands). The pulser (AVL-2-PS-P-4076, Avtech, Electro systems, Ottawa, ONT, Canada) transmitted an electrical pulse consisting of a half cycle of $80 \mathrm{~V}$ amplitude. The transducer transmitted a series of US detection pulses onto the vibrating target. The reflected pulses were low-pass filtered and amplified (5052 PR, Panametrics, Zoersel, Belgium) and then digitised with an oscilloscope (9400 LeCroy, Geneva, Switzerland). The echoes are sampled at $100 \mathrm{MHz}$ rate. The oscilloscope was operated in sequence mode to acquire the 62 subsequent echoes, which were then recorded on a computer and imported in Matlab software for further analyses. The radiofrequency (RF) signal was resampled at $50 \mathrm{GHz}$. The 61 subsequent echoes were correlated with the first echo to estimate their time delays and deduce the displacement of the shaker.

Examples of recorded echoes are given in Fig. 3. The figure shows the time shift between two echoes (numbers 14 and 27) of the moving target. The resulting time values were converted to position values and plotted on the corresponding time scale. The measured values were compared with the readings of the strain gauge sensor to assess the validity and accuracy of the US technique.

\section{In vivo setup}

The sensitivity and accuracy of the US system is considered to be sufficient with respect to the foreseen 


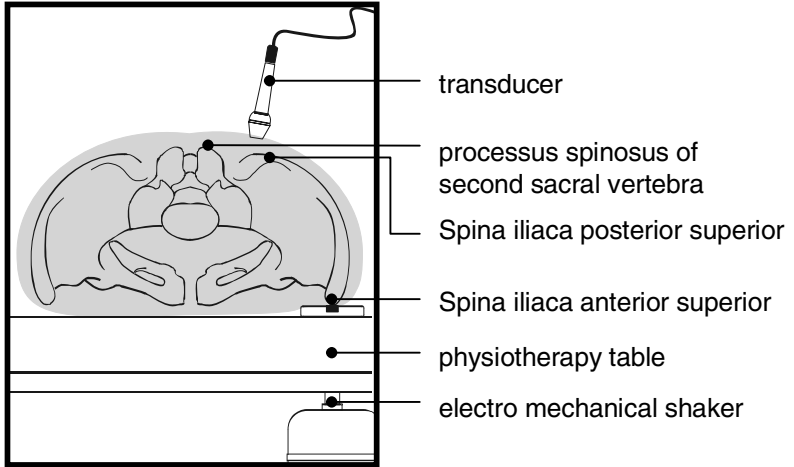

Fig. 4. Schematic representation of the in vivo setup.

working range of $(0.1$ up to $100 \mu \mathrm{m})$ to justify the application of this method for further in vivo measurements. However, in vivo experiments pose different challenges than do in vitro experiments. To gain insight into problems in an in vivo setup (Fig. 4) and to assess the feasibility of the method, we performed a number of pilot in vivo measurements on volunteers. The subject lay in prone position on a physiotherapy table, the left spina iliaca anterior superior (SIAS) was supported by a plastic disc protruding through the table. The plastic disc was connected to the rod of a shaker (model 200, Ling Dynamic Systems, Royston, England). An accelerometer was attached to the plastic disc to monitor the applied vibration amplitudes and waveforms. The ilium was excited with a range of amplitudes $(3 \mu \mathrm{m}-100 \mu \mathrm{m})$ at two different frequencies $(100 \mathrm{~Hz}$ and $200 \mathrm{~Hz})$. In detecting the vibrations of the ilium and the sacrum, we positioned the transducer ( $5 \mathrm{MHz}$, Aerotech, Agfa) respectively at the spina iliaca posterior superior (SIPS) and on the line through the left and right SIPS near the processus spinosus of second sacral vertebra. The transducer transmitted 62 pulses and the recorded echoes were processed as described previously.

\section{RESULTS}

Figure 5 shows an example of the measured displacement as a function of time. The piezo shaker was vibrating at $180 \mathrm{~Hz}$ with displacement amplitude of $2.5 \mu \mathrm{m}$. The measured displacement curve as calculated using time-delay measurements reproduces adequately both amplitude and frequency of the vibrations.

To estimate the accuracy of the US measurement system with respect to the strain gauge position sensor, we analysed a total of 66 measurement results obtained by changing the displacement amplitude and the frequency of the piezo shaker vibrations. The results are shown in Fig. 6. Figure 6a displays the scatterplot of the US measurement results against the strain gauge sensor

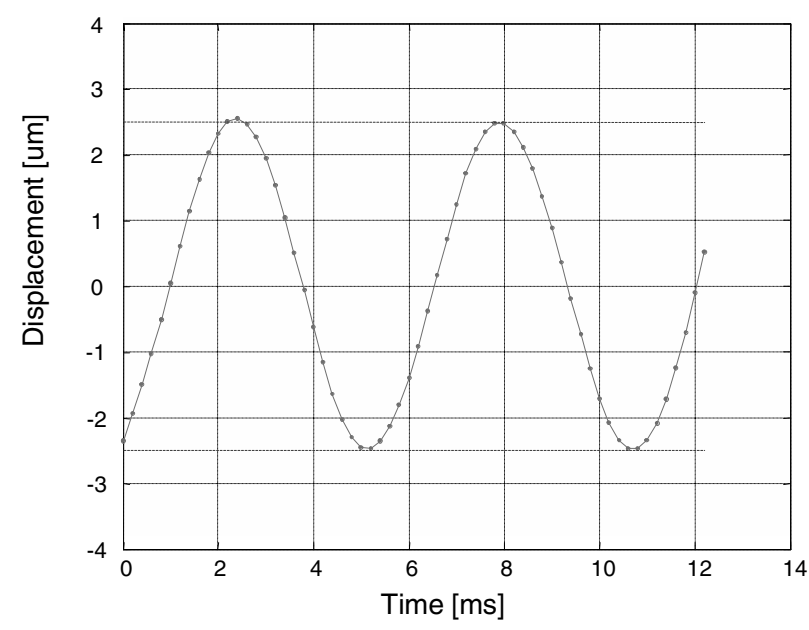

Fig. 5. Position time plot of a shaker, vibrating at $180 \mathrm{~Hz}$ and $5 \mu \mathrm{m}$ displacement, as detected with a $5-\mathrm{MHz}$ transducer.

readings. A least square fit resulted in $\mathrm{R}^{2}=0.999$, and the regression line can be given by $u=1044 * 10^{-2}+$ $0.991 x$, where $x$ is the strain gauge value and $u$ the US displacement value. This demonstrates a very good agreement with the sensor output, therefore validating the experimental procedure.

The relative error of the US measurements is assumed to have a normal distribution with mean $-0.1 \%$ and SD $3.65 \%$. For the relevant range of vibrations $(0.25$ to $3 \mu \mathrm{m})$, we can conclude that $95 \%$ of the measured amplitudes obtained with the US system may differ by less than $\pm 7 \%$ from the strain gauge values. However, the error in terms of percentages increases with decreasing amplitude (Fig. 6b). The maximum difference between frequencies measured by the US system and the reference values was $4.4 \%$.

In vivo, the US system was able to detect bone vibrations down to submicrometer level. Figure 7 shows the displacement measured on the dorsal side of the ilium while excited at the ventral side. Although this result is very preliminary, it shows that the dynamic response (i.e., a vibration of $0.5-\mu \mathrm{m}$ amplitude) of the ilium can be detected when a low-frequency vibration is applied to the pelvic girdle at a different location.

\section{DISCUSSION}

Ultrasound with a frequency of $5 \mathrm{MHz}$ has a wavelength of $0.3 \mathrm{~mm}$. Using the phase of the reflected signal, an accurate position, down to $1 \mu \mathrm{m}$ or less, of the reflector can be measured as shown in this study. If our identification method described in the introduction requires higher accuracy, then further improvements using signal processing and US parameter detection can be applied.

The amplitude measurement error of the US system increases with decreasing amplitude and increasing fre- 


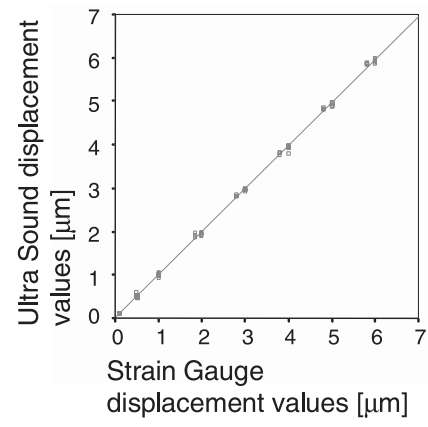

a)

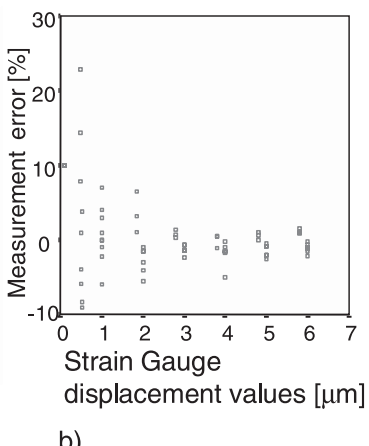

b)

Fig. 6. (a) Scatterplot of the US displacement measurement results against the strain gauge sensor values. (b) Scatterplot of the measurement error against the amplitude, demonstrating that the error decreases with increasing amplitude.

quency of the measured vibration as a consequence of the signal-to-noise ratio and pulse repetition frequency. From our data set, we can conclude that the lowest measurable amplitude is below $1 \mu \mathrm{m}$. The frequency measurement error can be explained by the number of measurements, which is 62 for the described measurement setup.

The anticipated working range (submicrometer amplitudes) required a high precision reference measurement system to estimate the accuracy of our US system. The strain gauge sensor, which is used as a reference measurement system, is an integral part of the applied piezo shaker and has a resolution better than $1 \mathrm{~nm}$.

To obtain reproducible measurements on one subject in vivo, provisions will have to be taken. For example, because the measurement method is based on relative displacement of the US reflector (the bone) with respect to the transducer, the influence of all movements of either the subject or the operator must be excluded.

It is intended to identify the biomechanical properties of the sacroiliac joints using common model updating tools (in modal, time or frequency domain). A first sensitivity-based identification method in modal domain was developed by one of the authors (Conza and Rixen 2004). The parameters considered in this identification method are the 11 stiffness coefficients of the pelvic ligaments and the 3 masses of the rigid bodies, the pelvic bones. The method was not able to identify parameters in presence of noise. A second identification method, in frequency domain, is now under development, which aims at the minimisation of an objective, function rendering the difference between simulated noise-affected frequency response function (FRF) measurements and analytical FRFs generated during model updating iterations. The real measurements will replace the simulated FRF in the developed postprocessing algorithm. The identification uses the displacements as measured at ac-

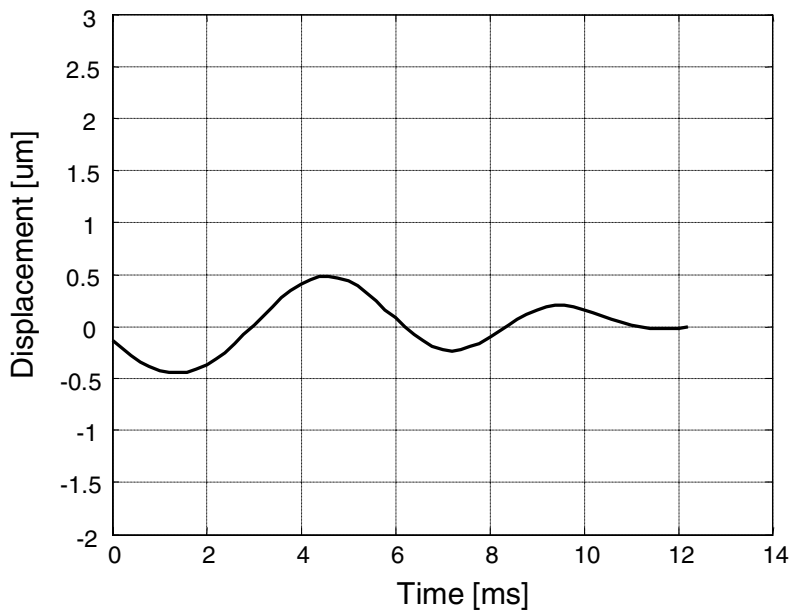

Fig. 7. Position-time plot of a vibrating ilium resulting from an applied vibration of $200 \mathrm{~Hz}$ and $3 \mu \mathrm{m}$ amplitude.

cessible landmarks on the pelvis. Measurement of the vibrations of the pelvic bones simultaneously (or at a fixed delay between subsequent measurements) at different locations allows the use of phase difference as an input parameter for the identification algorithm. This will provide us highly relevant information to identify damping in the pelvic girdle. Focus on the development of a configuration in which two phase-locked transducers can be activated allows such a measurement protocol. However, successful measurements require a highly stable electronic system with respect to triggering and phase locking.

Based on preliminary computer simulations, the relevant information from the dynamic response is expected to be in the frequency range of 10 to $500 \mathrm{~Hz}$, and the lowest vibration requires a record length of $0.1 \mathrm{~s}$.

\section{CONCLUSION}

In this paper, a new noninvasive method to measure bone vibrations was presented. In vitro evaluation of the experimental procedure showed that displacement amplitudes as low as $0.25 \mu \mathrm{m}$ can be detected with a maximum error of $\pm 7 \%$. Preliminary volunteer tests have demonstrated the ability of the technique to detect low-frequency vibrations of the pelvic bones. The reproducibility will be the focus of further investigation.

From these preliminary results, it is concluded that this approach based on US waves can be used to develop a new measurement tool to study the relation between the biomechanical properties of the sacroiliac joints and low back pain.

Acknowledgements-This research was supported by the Technology Foundation STW, Applied Science Division of NWO and the Technology Programme of the Ministry of Economic affairs of the Netherlands. 


\section{REFERENCES}

Bickley L, Szilagyi P. Bates' guide to physical examination \& history taking. Philadelphia: Lippincott Williams \& Wilkins, 2002.

Buyruk HM, Stam HJ, Snijders CJ, et al. The use of color Doppler imaging for the assessment of sacroiliac joint stiffness: A study on embalmed human pelvises. Eur J Radiol 1995;21:112-116.

Conza NE, Rixen DJ. Dynamic model of the human pelvis-Parameter identification. In: Proceedings of the XXII International Modal Analysis Conference, Bethel, CT: Society for Experimental Mechanics, Inc. 2004.

de Korte CL, Schaar JA, Mastik F, Serruys PW, van der Steen AF. Intravascular elastography: from bench to bedside. J Interv Cardiol 2003; 16:253-259.

Dreyfuss P, Michaelsen M, Pauza K, McLarty J, Bogduk N. The value of medical history and physical examination in diagnosing sacroiliac joint pain. Spine 1996;21:2594-2602.

Ferrara K, DeAngelis G. Color flow mapping. Ultrasound Med Biol 1997;23:321-345.

Greene W, Heckman J. The clinical measurement of joint motion. Am Acad Orthop Surg 1994;1-14.

Hoppenfeld S. Physical examination of the spine and extremities. New York: Appleton-Century-Crofts, 1976.

Jacob HA, Kissling RO. The mobility of the sacroiliac joints in healthy volunteers between 20 and 50 years of age. Clin Biomech (Bristol, Avon) 1995;10:352-361.
Kovacs FM, Abraira V, Zamora J, et al. Correlation between pain, disability, and quality of life in patients with common low back pain. Spine 2004;29:206-210.

Mofidi A, Sedhom M, O'Shea K, et al. Screening of lower back pain, low back pain clinic. The clinical experience. Ir Med J 2003;96: $270-273$.

Ophir J, Cespedes I, Ponnekanti H, Yazdi Y, Li X. Elastography: A quantitative method for imaging the elasticity of biological tissues. Ultrason Imaging 1991;13:111-134.

Pool-Goudzwaard A, Hoek van Dijke G, Mulder P, et al. The iliolumbar ligament: Its influence on stability of the sacroiliac joint. Clin Biomech (Bristol, Avon) 2003;18:99-105.

Sims JA, Moorman SJ. The role of the iliolumbar ligament in low back pain. Med Hypotheses 1996;46:511-515.

Snijders C, Vleeming A, Stoeckart R. Transfer of lumbosacral load to iliac bones and legs, Part 1: Biomechanics of self-bracing of the sacroiliac joints and significance for treatment and exercise. Clin Biomech 1993;8:285-294.

Sturesson B, Selvik G, Uden A. Movements of the sacroiliac joints. A roentgen stereophotogrammetric analysis. Spine 1989;14:162-165.

van der Wurff P, Hagmeijer RH, Meyne W. Clinical tests of the sacroiliac joint. A systemic methodological review. Part 1: reliability. Man Ther 2000a;5:30-36.

van der Wurff P, Meyne W, Hagmeijer RH. Clinical tests of the sacroiliac joint. Man Ther 2000b;5:89-96. 